\title{
Retalho de Deslizamento Jigsaw Puzzle: Utilização nos Defeitos Cirúrgicos dos Membros
}

\section{Jigsaw Puzzle Advancement Flap: Application in Limbs Surgical Defects}

Ermelindo Tavares ${ }^{1}$

\section{RESUMO}

INTRODUÇÃO: O retalho de deslizamento jigsaw puzzle é utilizado geralmente no encerramento de defeitos cirúrgicos nasais, em particular da região alar. No entanto, outras localizações faciais e extra-faciais podem beneficiar do mesmo.

CASO CLÍNICO: Três doentes foram submetidos à exérese de carcinoma espinocelular do membro superior (dois casos) e inferior (um caso), seguido de reconstrução com retalho de deslizamento jigsaw puzzle. Foram obtidos bons resultados funcionais e estéticos nos três casos.

DISCUSSÃO: O retalho de deslizamento jigsaw puzzle permite encerrar defeitos faciais e extra-faciais de pequenas, médias e grandes dimensões, com resultados estéticos e funcionais semelhantes às restantes alternativas clássicas. CONCLUSÃO: Os resultados obtidos nos três casos demonstraram que retalho de deslizamento jigsaw puzzle é uma boa opção no encerramento de defeitos cirúrgicos localizados nos membros.

PALAVRAS-CHAVE: Extremidades; Neoplasias da Pele; Procedimentos Cirúrgicos Reconstrutivos; Retalhos Cirúrgicos 


\begin{abstract}
INTRODUCTION: The jigsaw puzzle advancement flap is generally used in the closure of nasal surgical defects, especially in the alar region. However, others facial and extra facial locations are able to be contemplated.

CASE REPORT: Three patients were submitted to wide excision of squamous cell carcinoma located in the upper (two cases) and lower (one case) limb, followed by jigsaw puzzle advancement flap. Good functional and aesthetic results were achieved in all cases.

DISCUSSION: Jigsaw puzzle advancement flap may be used in the reconstruction of small, medium and large facial and extra facial defects. The aesthetic and functional results are as good as in others classic surgical techniques.

CONCLUSION: The results achieved with these three cases allow us to state that JPAF is a good surgical option for skin defects located on the limbs.
\end{abstract}

KEYWORDS: Extremities; Reconstructive Surgical Procedures; Skin Neoplasms; Surgical Flaps

\section{INTRODUÇÃO}

O membro é uma localização comum de lesões pré-malignas (queratoses actínicas) e cancro cutâneo, sendo fatores predisponentes importantes para o aparecimento destas dermatoses, nesta e noutras regiões, a idade avançada (a partir dos 50 anos), o sexo masculino, a exposição aos raios ultravioletas (crónica, intermitente e intensa), os fotótipos cutâneos baixos (tipo I e II da classificação de Fitzpatrick), os fatores genéticos e a imunossupressão. O carcinoma basocelular e espinocelular são os tipos clínicos mais observados nos membros, seguido de melanoma. Os menos comuns são o carcinoma das células de Merkel, os sarcomas, as neoplasias malignas dos anexos, os linfomas, as leucemias/linfomas e as metástases. Apesar do declínio da taxa de mortalidade, a incidência dos tumores malignos da pele tem aumentado drasticamente, mesmo em idades jovens.

O encerramento das feridas cirúrgicas dos membros pode ser desafiante, em particular nos casos de fotoenvelhecimento cutâneo severo, defeitos de grandes dimensões, cicatrizes rádicas ou doutra etiologia, linfedema e dermatoses inflamatórias crónicas (psoríase, eczema). As opções cirúrgicas são variadas (sutura direta, cicatrização por segunda intenção, retalhos e enxertos). Em 2005, Goldberg et al ${ }^{1}$ descreveram o retalho de deslizamento jigsaw puzzle na reconstrução de defeitos laterais externos da asa do nariz. O mesmo foi aplicado por outros autores nas mesmas ${ }^{2,3}$ e noutras localizações da cabeça e pescoço. ${ }^{4}$

Neste artigo o autor propõe, através da apresentação de três casos clínicos, o encerramento de defeitos cirúrgicos dos membros com retalho de deslizamento jigsaw puzzle.

\section{DESCRIÇÃO DE CASO CASO 1}

Homem, caucasiano, agricultor, de 87 anos, anticoagulado com rivaroxabano por fibrilhação auricular crónica. Observado por nódulo cutâneo com 2,0cm, redondo, não ulcerado, móvel e indolor, localizado no dorso da mão direita (Fig.s 1a, 1b). Tinha evolução desconhecida. Não eram evidentes lesões satélites e em trânsito e adenopatias regionais palpáveis. Estabeleceu-se o diagnóstico clínico de carcinoma espinocelular e foi submetido à exérese cirúrgica.

\section{CASO 2}

Mulher, caucasiana, agricultora reformada, de 93 anos. Referenciada por placa cutânea dolorosa e ulcerada no dorso da mão esquerda (Fig.s 2a, 2b), com 1,0 por 1,5 cm e um ano de evolução. Não tinha lesões satélites e em trânsito e adenopatias regionais palpáveis. Colocou-se como hipótese de diagnóstico clínico carcinoma espinocelular e foi submetida à excisão cirúrgica.

\section{CASO 3}

Homem, caucasiano, de 78 anos, reformado, residente em Angola durante vários anos, com antecedentes pessoais de múltiplas queimaduras solares na adolescência e juventude, excisão de múltiplos carcinomas basocelulares (face, couro cabeludo e tórax) e espinocelulares (couro cabeludo), acidente vascular cerebral, patologia arterial periférica, demência vascular e hipertensão arterial essencial. Encontrava-se medicado com rivaroxabano. Recorreu à consulta por nódulo cutâneo redondo, ulcerado, com 2,5 cm, móvel e indolor, localizado no terço distal do joelho direito (Fig.s 3a, 3b). Tinha quatro meses de evolução. Não se observaram lesões satélites e em trânsito e não tinha adenopatias regionais palpáveis. Estabeleceu-se o diagnóstico clínico de carcinoma espinocelular e foi submetido ao tratamento cirúrgico. 

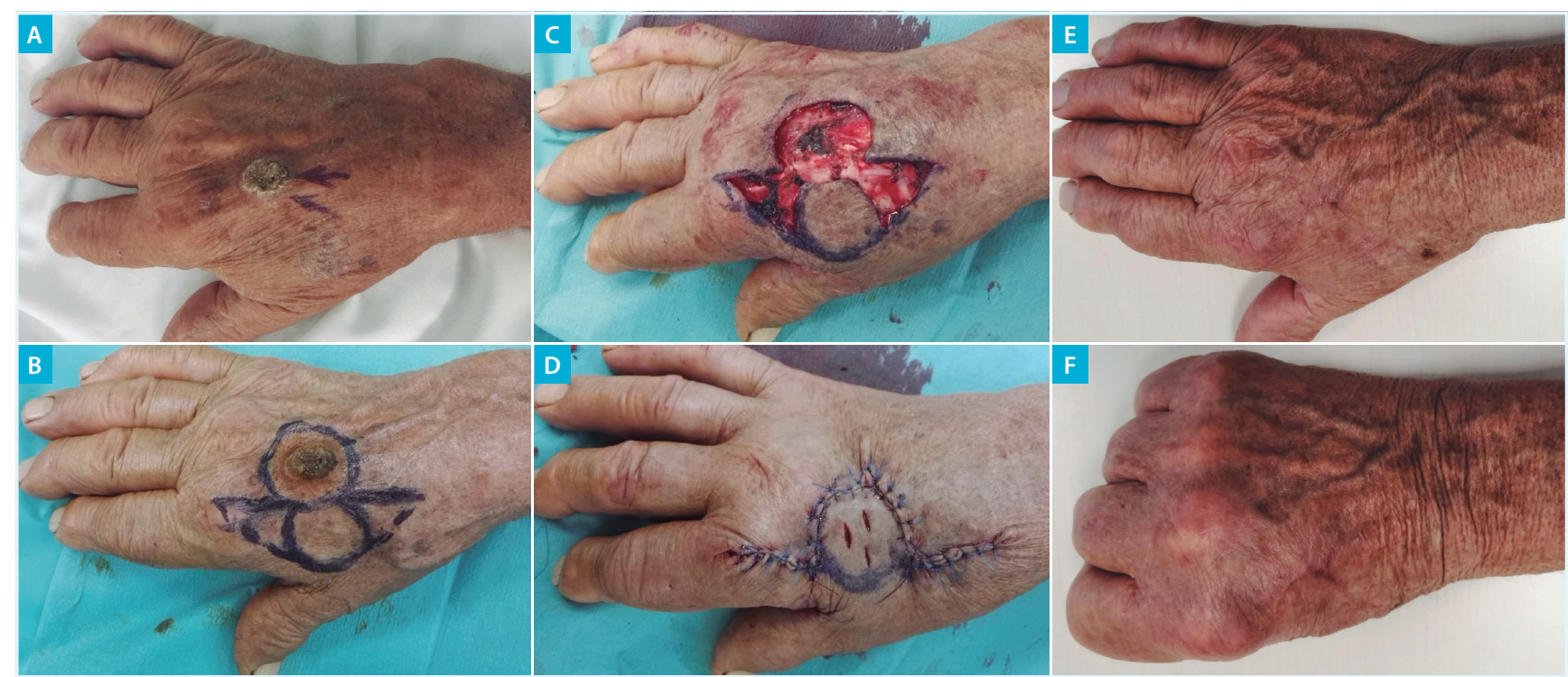

FIGURA 1. (CASO 1): Carcinoma espinocelular no dorso da mão direita (a). Retalho de deslizamento jigsaw puzzle delineado (configuração em "ómega") lateralmente ao defeito e dissecado (b, c). Resultado imediato com incisões no centro do retalho para evitar hematoma (d). Bom resultado estético e funcional após oito semanas, com cicatriz hipertrófica ligeira (e, f).
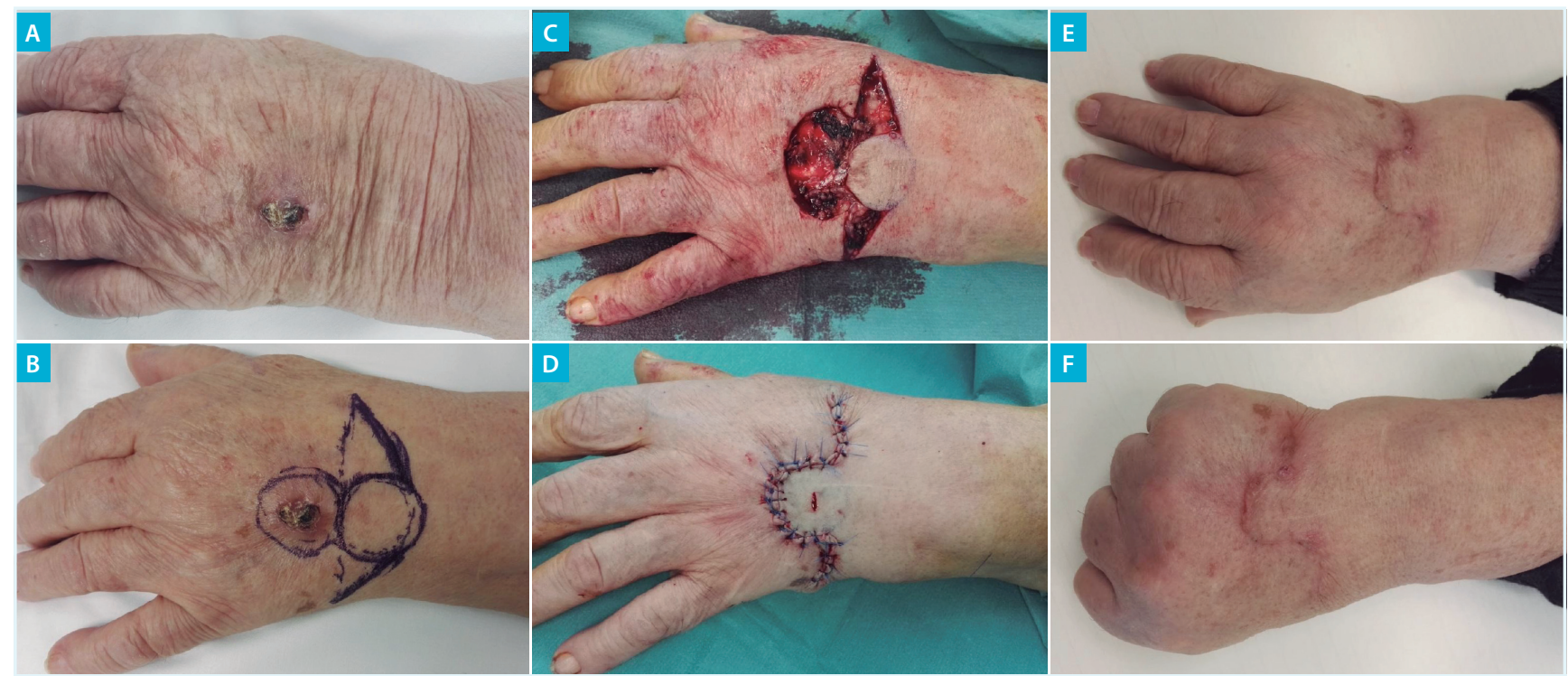

FIGURA 2. (CASO 2): Carcinoma espinocelular no dorso da mão esquerda (a). Retalho de deslizamento jigsaw puzzle delineado (configuração em "ómega") superiormente ao defeito e dissecado (b, c). Resultado imediato com incisão central (d). Bom resultado estético e funcional após seis semanas (e,f).

\section{TÉCNICA CIRÚRGICA}

O retalho jigsaw puzzle foi o método utilizado para a reconstrução cirúrgica. Todos foram operados sob anestesia local num tempo operatório. As neoplasias foram excisadas de forma circular (Fig.s 1b, 2b, 3b) e com margens laterais e profundas adequadas. O retalho foi delineado com configuração em "ómega" na posição lateral (caso 1), superior (caso 2) e inferior (caso 3) em relação ao defeito e com o mesmo diâmetro e espessura deste último (Fig.s 1b, 1c, 2b, 2c, 3b, 3c.). O encerramento interno foi realizado com fios de sutura absorvíveis e o externo com os não absorvíveis que foram removidos aos 10-12 dias do pós-operatório. A fim de evitar he- matomas e comprometimento dos retalhos, nos casos 1 e 2 foram efetuadas pequenas incisões com 3-5 mm de comprimento no centro do retalho (Fig.s 1d, 2d) e no caso 3 foi colocado dreno passivo durante 48 horas (Fig. 3d).

\section{RESULTADOS}

Não se registaram complicações no pós-operatório (hemorragia, isquemia, necrose, deiscência, infeção e hematoma). Os resultados estéticos e funcionais foram aceitáveis (Fig.s 1e, 1f, 2e, 2f, 3e). No caso 1 observou-se cicatriz hipertrófica ligeira (Fig.s 1e, 1f) que se resolveu completamente com modulador cicatricial tópico. 

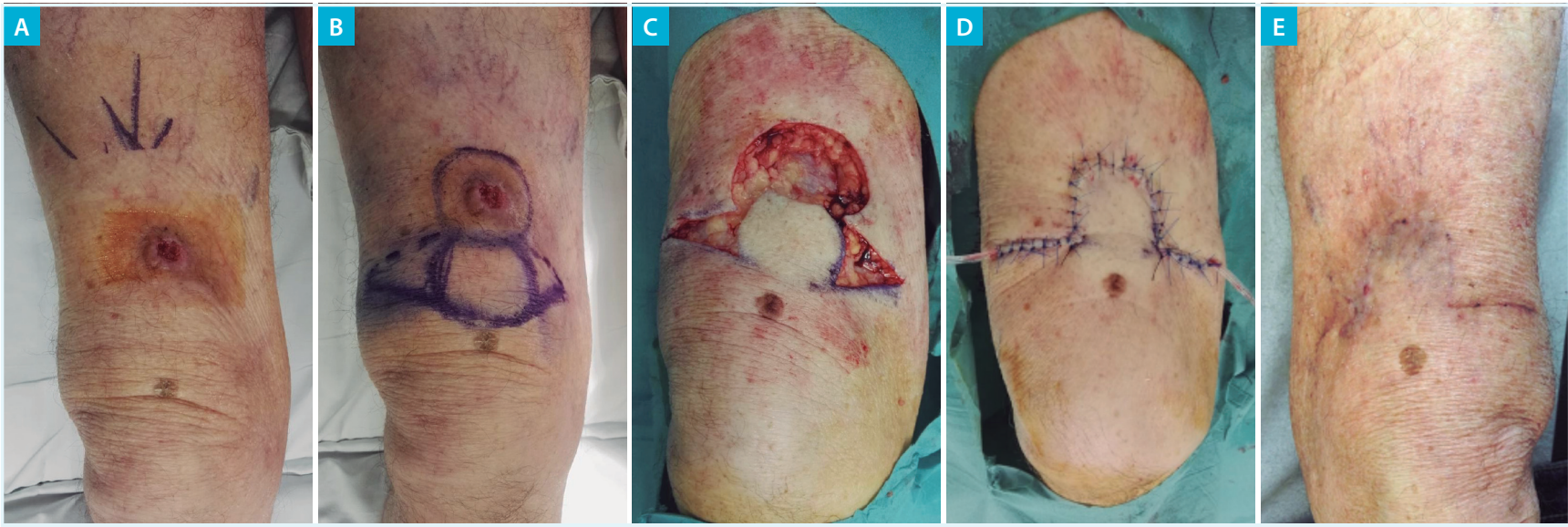

FIGURA 3. (CASO 3): Carcinoma espinocelular na face anterior do 1/3 distal da coxa direita coxa direita (a). Retalho de deslizamento jigsaw puzzle delineado (configuração em "ómega") inferiormente ao defeito e dissecado (b, c). Resultado imediato com colocação de dreno passivo (d). Bom resultado estético após oito semanas (e).

Nenhum caso desenvolveu efeito em alçapão. Nos casos 2 e 3 houve perda de seguimento por motivos não relacionados com a dermatose neoplásica, aos 12 e 18 meses, respetivamente. Ambos não tinham, até essa data, recidiva local, loco-regional ou à distância. O caso 1, após 3 anos, permanece em seguimento regular e sem recidivas.

\section{DISCUSSÃO}

O retalho de deslizamento é uma técnica cirúrgica que se baseia no movimento unidirecional (sem rotação ou transposição sobre um ponto pivô) de pele adjacente para o encerramento do defeito. Apresenta, geralmente, um pedículo aleatório. De acordo com alguns historiadores, trata-se duma técnica milenar provavelmente descrita e executada pela primeira vez por Aulus Cornelius Celsus no século I, na Roma antiga. Posteriormente foi aperfeiçoada por vários cirurgiões, em particular os franceses, em meados do século XIX, sob a designação lambeau par glissement (retalhos deslizantes). ${ }^{5} \mathrm{~A}$ proximidade permite manter a espessura, a cor e a textura cutânea e, desta forma, obter melhor resultado estético. A mobilidade da pele vizinha adjacente é uma das condições fundamentais na sua escolha e execução. Por apresentar grande versatilidade pode ser utilizado em inúmeras áreas cutâneas (face, pescoço, tronco e membros).

O retalho de avanço jigsaw puzzle foi descrito por Goldberg para a reconstrução de defeitos laterais da asa do nariz, sendo uma alternativa aos métodos clássicos, nomeadamente o retalho pediculado em ilha (avanço e rotação) e o nasogeniano de transposição. Em 2013, Alkalay et a ${ }^{4}$ relataram um caso onde o retalho de des- lizamento jigsaw puzzle-like foi utilizado com sucesso no encerramento dum defeito retroauricular com $20 \mathrm{~mm}$. Em 2017, Arpaci et alo utilizaram uma técnica semelhante para encerrar defeitos circulares da face, do tronco, das nádegas e das mãos. Foi designado retalho de deslizamento em "ómega" e executado de acordo com alguns princípios matemáticos estabelecidos pelos mesmos. Excetuando este último relato, são escassos na literatura os trabalhos científicos sobre aplicação do retalho de avanço jigsaw puzzle ou técnica análoga nos membros.

A ausência de elasticidade e mobilidade cutânea torna inexequível o retalho de deslizamento jigsaw puzzle nos membros, tal como acontece com os restantes retalhos de avanço noutras áreas anatómicas. Os idosos, classe etária onde predominam os cancros de pele, são ótimos candidatos por apresentarem grande flacidez cutânea secundária ao envelhecimento fisiológico ou fotoenveIhecimento. Perante uma pele pouco elástica e móvel por cicatrizes crónicas, radiodermites, linfedema ou outras etiologias, as melhores alternativas são os enxertos cutâneos (total ou parcial), cicatrização por segunda intenção e, nalguns casos, os retalhos de transposição.

O posicionamento do retalho em relação ao defeito é um ponto importante na execução do retalho jigsaw puzzle. Deve ser delineado lateralmente (interno, externo, superior ou inferior) e, sempre que possível, paralelo às linhas de relaxamento da pele (linhas de Langer). Desta forma, o deslizamento e a integração fazem-se sem tensão e as cicatrizes são colocadas nas pregas cutâneas, favorecendo melhor resultado estético. Neste trabalho, no caso 1 não foi possível respeitar este princípio devido à localização do tumor e, como tal, obteve-se como resultado estético uma cicatriz hipertrófica ligeira. 
No que diz respeito às complicações, como observado nos três casos relatados e em inúmeras publicações da região nasal, o efeito em alçapão e a necrose são raras, desde que os retalhos sejam elaborados com espessura adequada (não superior à do defeito) e pedículo largo. Os drenos passivos e/ou as pequenas incisões no centro do retalho minimizam o risco de hematoma. Ambos procedimentos são facultativos, mas, na opinião do autor, devem ser sempre equacionados nos casos de toma regular de antiagregantes e/ou anticoagulantes orais, mesmo após boa hemóstase. Neste artigo, dois doentes tratados estavam medicados com anticoagulantes orais.

Os exemplos de procedimentos alternativos para o encerramento de defeitos cirúrgicos dos membros incluem o retalho de avanço pediculado $V Y^{7}$, os retalhos de rotação, ${ }^{8,9}$ o retalho de avanço AT,10 o retalho bilobulado, ${ }^{11}$ o retalho pediculado em pedra angular, ${ }^{12,13} \mathrm{O}$ enxerto de pele total e a cicatrização por segunda intenção. Com exceção desta última que, no geral, resulta numa cicatriz hipertrófica central de grau variável, os resultados estéticos e funcionais obtidos com o retalho jigsaw puzzle são globalmente idênticos aos dos restantes procedimentos.

\section{CONCLUSÃO}

O retalho de deslizamento jigsaw puzzle apresenta grande versatilidade e, como tal, deve constituir mais um método a ter em consideração na reconstrução dalguns defeitos cirúrgicos de pequenas e médias dimensões nos membros.

\section{AGRADECIMENTOS/ ACKNOWLEDGEMENTS}

Serviço de Anatomia Patológica e equipa de enfermagem e auxiliares do Bloco Operatório Central e da Consulta Externa, Hospital Vila Franca de Xira.

CONFLITOS DE INTERESSE: Os autores declaram não ter qualquer conflito de interesse na realização do presente trabalho.

FONTES DE FINANCIAMENTO: Não houve qualquer fonte de financiamento na realização do presente trabalho.

CONFIDENCIALIDADE DOS DADOS: Os autores declaram ter seguido os protocolos da sua instituição acerca da publicação dos dados de doentes.

CONSENTIMENTO: Consentimento do doente para publicação obtido.
PROVENIÊNCIA E REVISÃO POR PARES: Não comissionado; revisão externa por pares.

CONFLICTS OF INTEREST: The authors declare that they have no conflicts of interest.

FINANCIAL SUPPORT: This work has not received any contribution, grant or scholarship.

CONFIDENTIALITY OF DATA: The authors declare that they have followed the protocols of their work center on the publication of data from patients.

PATIENT CONSENT: Consent for publication was obtained.

PROVENANCE AND PEER REVIEW: Not commissioned; externally peer reviewed.

\section{REFERÊNCIAS}

1. Goldberg LH, Kimyai-Asadi A, Silapunt S. "Jigsaw puzzle" advancement flap for repair of a surgical defect involving the lateral nasal ala. Dermatol Surg. 2005;31:569-71.

2. Sanches MM, Pinto Al, Filipe PL, Silva JM. "Jigsaw puzzle" advancement flap. J Cutan Aesthet Surg. 2018;11:248-9. doi: 10.4103/JCAS.JCAS_38_18.

3. da Cunha JA, Pessotti NS, Rocha CA, Maruyama FN. "Jigsaw puzzle" advancement flap: an unusual surgical technique for nasal alar reconstruction. Surg Cosmet Dermatol. 2019;11:234-37.

4. Alkalay R, Alcalay J. The "jigsaw puzzle" advancement flap for reconstruction of a retroauricular surgical defect. J Drugs Dermatol 2013;12:115-63.

5. Tenzel RR. Reconstruction of the central one half of an eyelid. Arch Ophthalmol. 1975;93:125-26.

6. Arpaci E, Altun S, Ertas NM, Uysal AC, Inozu E. Omega ( $\Omega$ ) advancement flap: a circular flap design for the aesthetic closure of circular skin defect. Aesthetic Plast Surg. 2017;41:327-34. doi: 10.1007/s00266-016-0751-5.

7. Tavares E, Castellano DP, Rosa J. Retalho de avanço V-Y de pedículo proximal para encerramento de defeito cirúrgico do dorso da mão. Rev Soc Port Dermatol Venereol. 2012;70:34547.

8. Cardoso PM, Santos P, Azevedo F. Rotation flap for closure of surgical defect on the dorsum of the hand. Surg Cosmet Dermatol. 2011;3:348-49.

9. Cecchi R, Bartoli L, Brunetti L. Double helix flaps for lower leg defects: report of 4 cases. J Cutan Aesthet Surg. 2013;6:16465. doi: 10.4103/0974-2077.118425.

10. Pontello Júnior R, Kondo RN, Pontello R. The uses of the AT flap for reconstructing surgical wounds on the dorsum of the hand. Surg Cosmet Dermatol. 2013;5:27072.

11. Tavares, E. Reconstrução do dorso da mão com retalho bilobulado: uma opção cirúrgica incomum. Gazeta Méd. 2018;5:12425.

12. Hu M, Bordeaux JS. The keystone flap for lower extremity defects. Dermatol Surg. 2012;38:490-93. doi: 10.1111/j. 1524-4725.2011.02236.x.

13. Rao AL, Janna RK. Keystone flap: versatile flap for reconstruction of limb defects. J Clin Diagn Res. 2015;9:PC05-7. 